\title{
Design of Polymorphic Network Architecture Based on Integrated Operation Gateway
}

\author{
Ligang $\mathrm{Li}^{1,3, \mathrm{a}}$, Jinchao $\mathrm{Xiao}^{2, \mathrm{~b}}$, Zhongfeng Wang ${ }^{1,3, \mathrm{c}}$, Jianlong Huang ${ }^{1,3, \mathrm{~d}}$ \\ ${ }^{1}$ Shenyang Institute of Automation, Chinese Academy of Sciences, Shenyang,china 110016 \\ ${ }^{2}$ Shenyang Institute of Automation, Guangzhou, Chinese Academy of Sciences, Guangzhou,china \\ 511458 \\ ${ }^{3}$ Lab. of Networked Control Systems, Chinese Academy of Sciences, Shenyang,china 110016 \\ alilg@sia.cn, ${ }^{b} x i a o j i n c h a o @ s i a . c n,{ }^{c} w z f @ s i a . c n,{ }^{d}$ huangjianlong@sia.cn
}

\begin{abstract}
Keywords: Polymorphic, Integrated gateway, Monitoring networks
Abstract. In order to meet the diverse needs of equipment on-line monitoring, process monitoring in process industry, this paper designs a polymorphic network architecture based on an integrated gateway. It can easily adjust the network operation mode according to the change of application requirements. It provides users with a unified monitoring platform which facilitates the integration of wireless monitoring network based on different technologies.
\end{abstract}

\section{Introduction}

With the continuous development in the field of industrial applications, wireless communication technology is facing many new problems. The diverse needs of the various industrial processes require that the wireless monitoring network be polymorphic. In order to meet the different needs of equipment on-line monitoring, process monitoring, wireless monitoring network has great difference in network topology, access control, transmission mode, transmission medium and even transmission protocol. Among them, the network topology includes the star network, the tree network and the Mesh network; And the access control methods include CSMA, TDMA and FDMA and so on; In the aspect of transmission mode, we should consider not only the periodic real-time data, but also the non periodic burst data; In terms of transmission medium, we need to determine the use of low-frequency or high-frequency bands; And The use of standard or private protocols makes the transport protocol different. In the field equipment monitoring applications, several or dozens of nodes can form an online monitoring network. It can meet the application requirements using low-cost star topology for this small-scale monitoring network; For the wide area production process monitoring applications, because of the large number of measurement points, wide geographical distribution, real-time and high reliability requirements, the size of the monitoring network can reach 100 nodes or even thousands of nodes, and the network structure is complex. In summary, the process-oriented industrial monitoring and control system can be small-scale star topology network or large-scale tree topology or Mesh topology network. Real-time periodic data, burst data, non-real-time data transmission are mixed together in the same monitoring network.

In view of this situation, this paper designs a polymorphic network architecture based on an integrated gateway. It can easily adjust the network operation mode according to the change of application requirements. It provides users with a unified monitoring platform which facilitates the integration of wireless monitoring network based on different technologies.

\section{Polymorphic network architecture design}

The polymorphic network architecture designed in this paper is shown in Fig. 1, which is based on the integrated gateway. The upper layer of the architecture is the unified configuration management platform. The bottom layer is the polymorphic wireless monitoring and control network. The unified configuration management platform can support the polymorphic network plug-and-play function in the process industry intelligentization based on the unified description and analysis technology of information and equipment, provides the heterogeneous information representation method and 
support the different message formats of the underlying monitoring network. The platform can manage and configure the polymorphic network, and allocate network resources. The underlying wireless monitoring and control network accesses the unified configuration and management platform through the integrated gateway. The gateway, which communicates with different monitoring networks through a dedicated communication module, supports star network, tree network, mesh network and hybrid network. Coexistence of multiple monitoring networks is achieved by unified coordination and scheduling.

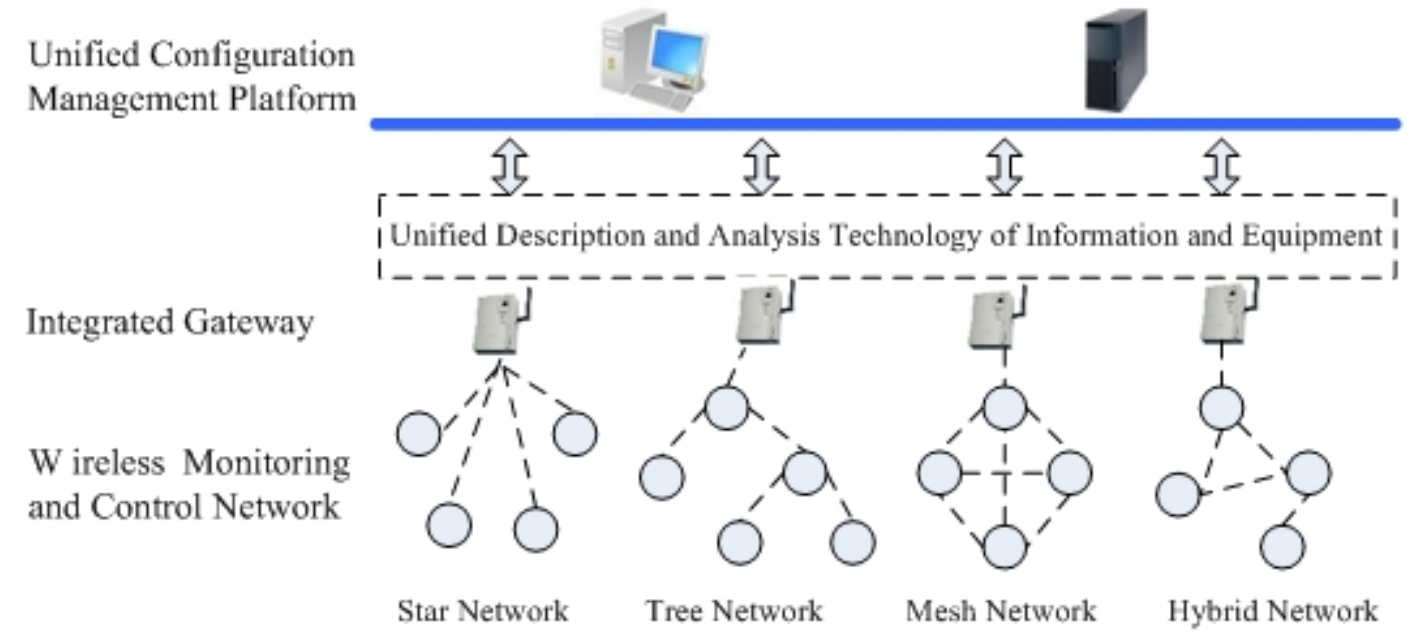

Figure 1: Polymorphic network architecture

\section{Integrated Gateway functional design}

The integrated gateway with the function of communication and intelligent management has standard data communication interface and physical interface, that is compatible with the existing measurement and control system, can realize the physical connection and the interaction between heterogeneous networks. Its bottom layer supports $433 \mathrm{MHz}, 470 \mathrm{MHz}, 2.4 \mathrm{GHz}$ and other radio frequency bands, and supports external interfaces such as Ethernet, RS232, and RS485.

According to the functional division, the integrated gateway is divided into 9 modules, as shown in Fig. 2: they are radio module, Profibus module, Modbus RTU module, Modbus TCP module, OPC_UA module, EtherNet/IP module, WIFI module, 3G module, Test\&Management module. These modules are used to implement bus protocol conversion, wireless network data forwarding and self management functions.

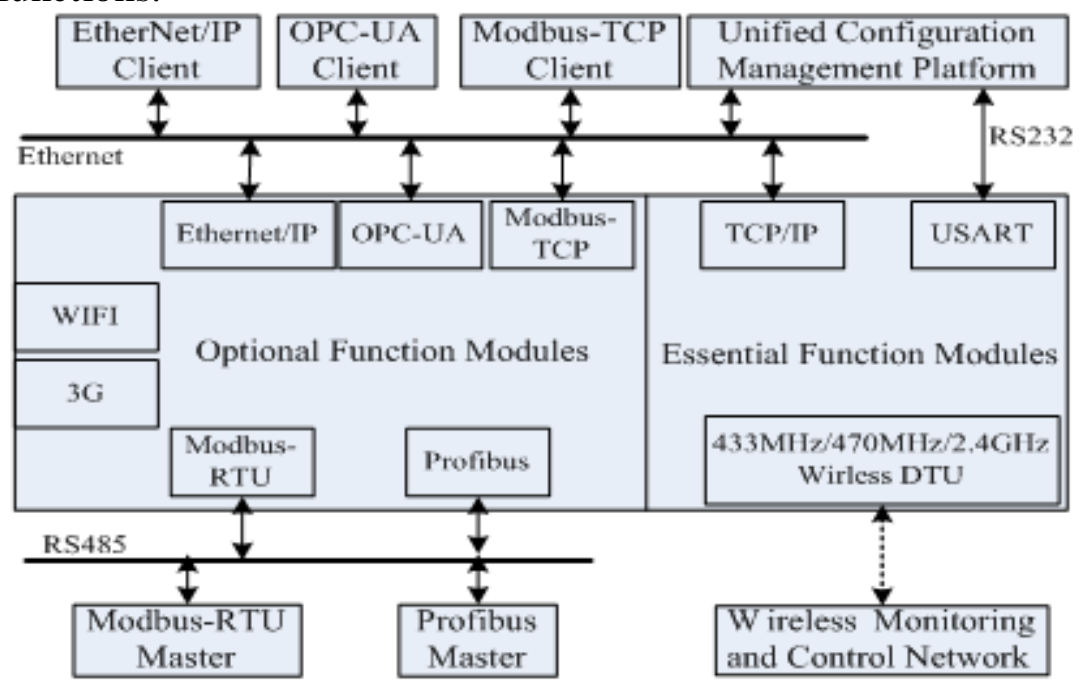

Figure 2: Integrated Gateway functional block diagram

Bus protocol conversion. To be applied to different application environments, the integrated gateway has the following functions: 1) Modbus-RTU slave function 2) Modbus-TCP server function 3) Profibus slave function 4) TCP / IP server function 5) EtherNet/IP slave function 6) OPC-UA 
server function. To this end, the integrated gateway provides a wide range of controller interfaces and protocol types. The gateway supports Modbus RTU / TCP, Profibus, EtherNet/IP and OPC-UA. Because the gateway has the protocol conversion function, so the data on different buses can be exchanged.

Wireless network data forwarding functions. The gateway supports $2.4 \mathrm{GHz}, 433 \mathrm{MHz}$, and 470 $\mathrm{MHz}$ wireless data modules that can be used to establish wireless monitoring networks with different topologies by which Wireless instruments and control systems can be linked to the gateway to meet industrial control requirements. In addition, the gateway supports standard WIFI networks and 3G networks, so that the gateway with remote access and management capabilities, greatly expand the flexibility of the gateway access to the background system.

The wireless data forwarding function includes data exchange between the wireless data transmission module and each bus agent, and also the exchange of data and instructions between the gateway and the unified management platform. The wireless network can be viewed as a configurable transparent channel through which the data on the wired and wireless networks can be interchanged. Data on the wireless network includes data acquisition, wireless network configuration instructions, wireless network node status, and so on.

Management functions. The management function is an interface that interacts with the unified management platform. It accepts directives from the unified management platform and responds, so that the unified management platform can monitor and control the operation of the whole wireless monitoring network. It has a unified data server interface for industrial control software to read gateway data and issue instructions to enable or disable certain interface function modules; configure the functional module of the basic operating parameters; provide equipment location, power and sensor working status information. The unified management platform can read the information of the gateway and wireless monitoring equipment from the gateway, including the running time, working interface module, ID, user remarks of the gateway, the running time, power supply capacity, data rate, device ID, connection information, sensor type of the wireless monitoring equipment. The gateway maintains this information through a management information base.

\section{Integrated gateway hardware design}

Integrated gateway hardware functional modules can be divided into power management, data processing, wireless communication and protocol conversion. The hardware ports related to Protocol conversion include: Ethernet, RS232 interface, RS485 interface and CAN interface. The main processor of the gateway selects the high performance ARM CORTEX-M4 processor STM32F407IGT6 of ST, and in order to ensure the implementation of OPC-UA and other protocols, the SDRAM and FLASH of 16MB and 128MB are extended. The hardware design is shown in Fig. 3.

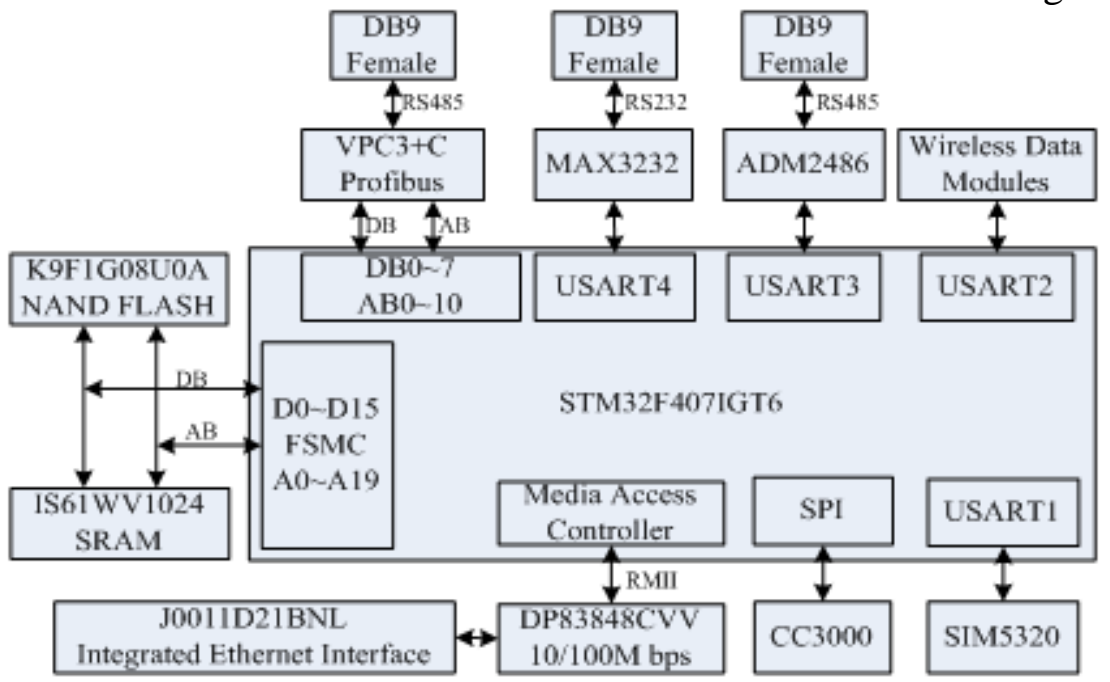

Figure 3: Hardware architecture 
The integrated gateway uses VPC $3+\mathrm{C}$ protocol chip and RS485 isolation chip together to constitute the Profibus DP hardware. MCU access the VPC3+C chip through the IO ports which emulate the address bus and data bus of the profibus. The integrated gateway uses the DP83848C and the internal MAC functions of the MCU to constitute the Fast Ethernet interface. The connection between DP83848C and MCU uses RMII, which takes up only a small amount of I/O port.RS485 function is realized by ADM2486 connected to USART1, and RS232 function is realized by MAX3232 connected to USART1. The gateway accesses $2.4 \mathrm{GHz}, 433 \mathrm{MHz}$ or $470 \mathrm{MHz}$ wireless network by a wireless data transmission module connected to USART2 of the MCU. At the same time it accesses WIFI network by a cc3000 module connected to SPI port of the MCU. The $3 \mathrm{G}$ network access function is achieved by a sim5320 module connected to USART1, and the sim5320 module can be configured by AT command.

The gateway power supply system consists of two DC 5V and one DC 3.3V power supplys, including one isolated DC 5V power supply.

External interfaces need to meet the TCP/IP, OPC-UA, Modbus-TCP, Profibus, Modbus-RTU, and other protocol communications. There are two types of external interfaces: RJ45 for TCP/IP, OPC-UA, Modbus-TCP, and DB9 for the Profibus, Modbus-RTU.

\section{Summary}

This paper designs a polymorphic network architecture based on an integrated gateway. It can easily adjust the network operation mode according to the change of application requirements. It provides users with a unified monitoring platform which facilitates the integration of wireless monitoring network based on different technologies.

\section{Acknowledgement}

In this paper, the research was sponsored by the National High Technology Research and Development Program of China(863 Pro.)(Project No. 2014AA041801). This work was supported by the Lab. of Networked Control Systems.

\section{References}

[1] IEC62601 Ed.1.0[S].WIA-PA communication network and communication profile, 2009.

[2] Liang Wei, Zhang Xiaoling. WIA-PA: industrial wireless network for process automation system architecture and communication protocol of [J]. Instrument standardization and metrology, 2009 (2).30 36.

[3] Fang yuanbai. Comments ON Application and Development of Wireless Technology in Process Industry [J].Process Automation Instrumentation Vol.34 No.2 February 2013 